・研究报告・

\title{
三江源地区雪豹保护优先区规划
}

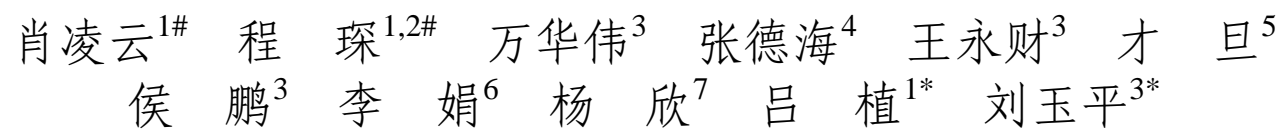

1 (北京大学生命科学学院自然保护与社会发展研究中心, 北京 100871)

2 (山水自然保护中心, 北京 100871)

3 (生态环境部卫星环境应用中心, 北京 100094)

4 (三江源国家公园管理局, 西宁 810001)

5 (青海省玉树藏族自治州林业局, 青海玉树 815000)

6 (University of California, Berkeley, CA 94720-3114, USA)

7 (四川省绿色江河环境保护促进会, 成都 610041)

摘要: 为了将有限资源合理投放到关键区域, 实现物种保护成效的最大化, 找出质量最好的栖息地及它们之间的 迁徙通道是制定保护规划的第一步。本研究以三江源的雪豹(Panthera uncia)栖息地为对象, 基于野外调查数据和 高分辨率卫星遥感数据, 利用物种分布模型、保护规划模型和连通度分析工具, 找出了三江源地区雪豹的核心栖 息地分布和潜在迁徙通道位置，分析了目前保护中的潜在威胁，并提出了针对三江源西、中、东三块区域的不同 保护对策。结果表明: (1)三江源西部核心栖息地比较小而破碎, 但迁徙通道较多且没有明显窄点, 未来应关注青藏 线的潜在阻碍作用，同时应防范道路沿线的野生动物盗猎; (2)中部区域横跨玉树-杂多-囊谦的雪豹栖息地是三江 源最大的核心雪豹栖息地, 在连通其他种群中也处于中心地位, 应通过种群监测确定其健康稳定, 对开发、偷猎等 威胁防微杜渐, 保持其源种群的作用; (3)东部区域人口密度高, 受人类活动的影响最大, 需保证阿尼玛卿、年保玉 则两块核心栖息地的质量, 并重点监测甘德县境内的省道处雪豹的迁徙通道是否畅通。三江源地区雪豹栖息地总 体质量较好, 建议将维持核心源种群的稳定性, 保持种群间迁徙通道的畅通作为三江源的雪豹景观保护工作的整 体目标。未来应充分利用天地一体化监测手段, 开展重要保护物种栖息地状况的评估和预警, 无其是非保护地区 域物种核心栖息地的开发建设活动。

关键词：雪豹; 栖息地; 高分辨率卫星; 保护优先区; 迁徙通道

\section{Defining conservation priority areas of snow leopard habitat in the Sanjiangyuan Region}

Lingyun Xiao ${ }^{1 \#}$, Chen Cheng ${ }^{1,2 \#}$, Huawei Wan ${ }^{3}$, Dehai Zhang ${ }^{4}$, Yongcai Wang ${ }^{3}$, Tsedan ${ }^{5}$, Peng $\mathrm{Hou}^{3}$, Juan $\mathrm{Li}^{6}$, Xin Yang ${ }^{7}$, Zhi Lü̈ ${ }^{*}$, Yuping Liu ${ }^{3 *}$

1 Center for Nature and Society, College of Life Sciences, Peking University, Beijing 100871

2 Shan Shui Conservation Center, Beijing 100871

3 Center for Satellite Application on Ecology and Environment, Ministry of Ecology and Environment, Beijing 100094

4 Management Bureau of the Three-River-Source National Park, Xining 810001

5 Forestry Bureau of Yushu Prefecture, Yushu, Qinghai 815000

6 Department of Environmental Science, Policy and Management, University of California, Berkeley, CA 94720-3114, USA

7 Sichuan Green River Environmental Protection Promotion Association, Chengdu 610041

Abstract: To maximize species conservation outcomes and effectively allocate limited resources, the first step in conservation planning is to define conservation priority areas and migration pathways between them. Based on field survey and high-resolution satellite data, we used species distribution models, conservation

收稿日期: 2019-01-18; 接受日期: 2019-05-23

基金项目: 国家重点研发计划(2018YFC0507200)、高分重大科技专项(05-Y30B02-9001-13/15)和国家青年科学基金(41801366)

\# 共同第一作者 Co-first authors

* 共同通讯作者 Co-authors for correspondence. E-mail: luzhi@pku.edu.cn; lyping3349@sina.com 
planning tools and connectivity analysis tools to define the core habitat area and potential corridors for snow leopards (Panthera uncia) in the Sanjiangyuan Region. With potential threats identified, we proposed differentiated conservation strategies for different parts of the Sanjiangyuan Region: (1) In the western region, snow leopard core habitats are mostly small and fragmented but with widespread migration pathways and no obvious bottlenecks. However, the Qinghai-Tibet Railway is a potential barrier for snow leopard populations in the western region and needs further investigation. (2) The central region contains the largest core habitat which also has highest centrality in the connectivity analysis. To maintain the function of these source populations, we recommend long-term monitoring, anti-poaching patrol and supervision on development projects for this region. (3) For the eastern region, which has the highest human density, it is important to safeguard two core habitats (Anemaqen and Nanpo Yutze) and maintain the narrow corridors in between them. The provincial highway in Gande County may be a potential barrier for snow leopard migration and deserves further monitoring. As the epicenter for snow leopard conservation in China, the habitat quality in the Sanjiangyuan Region remains good overall. The goal of snow leopard landscape conservation in Sanjiangyuan should be to maintain core source populations while keeping migration routes unblocked. Integrated space-ground monitoring approaches should be implemented in the future for assessments and early warning signs of habitat degradation for these important protected species, especially in non-protected areas in core habitat at risk for development.

Key words: snow leopard; habitat; high-resolution satellite data; conservation priority; dispersal corridor

在物种保护工作中, 为了将有限的资源合理投 放到不同区域并产生最大化的保护成果, 首先需要 对栖息地质量和其所面临的威胁进行评估, 找出核 心栖息地及其之间的迁徙通道，并在未来的保护规 划中维持核心栖息地的质量并保证通道畅通。大型 食肉动物能量需求高, 家域面积大, 对栖息地的需 求高, 繁殖相对缓慢, 种群密度低, 与人类的冲突 激烈, 导致它们在各类威胁面前极为脆弱(Carbone et al, 1999; Cardillo et al, 2004, 2005)。全球现存的31 种大型食肉动物中, 19种被IUCN列为受胁(包括易 危、濒危和极危)物种, 面临全球性或区域性灭绝的 风险; 24 种的种群数量持续下降; 能查出历史分布 范围的17种中, 当前的分布面积平均仅是历史分布 面积的47\% (Ripple et al, 2014)。

雪豹(Panthera uncia)起源于青藏高原腹地, 随 后扩散到中亚各大山系(Davis et al, 2010; Deng et al, 2011), 其栖息地与畜牧业分布区、藏传佛教分布 区、亚洲重要水源地高度重叠, 是亚洲山地生态系 统的旗舰种与伞护种。雪豹被IUCN红色名录评为 “易危”, 全球种群数量7,463-7,980 只 (McCarthy et al, 2017)。Snow Leopard Survival Strategy一书中全 面评估了雪豹面临的主要威胁(McCarthy \& Chapron, 2003): (1)家畜的竞争、栖息地退化和猎物短缺; （2)人类的报复性猎杀; (3)非法贸易。除此之外, 还 包括气候变化、开矿、基础设施建设(道路围栏等) 形成的阻碍(Jackson et al, 2013)。IUCN最新评级中
认为雪豹种群在过去 22.62 年(3个世代)内下降了 10\%, 也是基于有些地区不断增长的家畜、不断加 强的开矿和道路修建等压力来评估的(McCarthy et al, 2017)。

三江源地处青藏高原东部, 人类干扰较小, 猎 物种群丰富, 栖息地质量高, 是中国乃至世界雪豹 保护最有希望的地区之一。弄清三江源雪豹栖息地 的分布格局, 以及核心栖息地和迁徙通道的位置, 是制定细致的雪豹保护规划的首要需求。除了自然 环境变量如地形、气候等以外, 人类活动的分布对 雪豹栖息地的分布也存在不可忽略的影响。然而对 于雪豹栖息地的分析预测，长期以来受限于缺乏高 精度的人类活动分布数据。

本文采用高分辨率卫星数据解译的人类活动 斑块数据, 利用最大熵模型(maximum entropy modelling, Phillips et al, 2006)和Zonation保护规划模型 (Lehtomäki \& Moilanen, 2013)评估三江源雪豹栖息 地分布, 并对栖息地质量进行排序, 应用基于电路 理论设计的ArcGIS插件Linkage mapper (V1.10)计算 核心栖息地斑块间的雪豹栖息地连通度，并分析人 类活动斑块对连通度的可能影响, 以期为三江源地 区的雪豹保护规划制定提供基础数据。

\section{1 研究方法}

\section{1 研究区域}

三江源地区位于我国青海省南部，行政区域上 
包括海南、黄南、玉树、果洛 4 个州的 16 个县以及 格尔木市的唐古拉山镇, 总面积为 36.3 万 $\mathrm{km}^{2}$ 。该地 区以山地地貌为主, 山脉主要包括东昆仑山、阿尼 玛卿山、巴颜喀拉山和唐古拉山等, 海拔 3,335-6,564 m。三江源还是长江、黄河、澜沧江的 源头汇水区, 为全世界 $1 / 3$ 的人口提供水源 (三江源 自然保护区生态环境编辑委员会, 2002)。该地区属 典型的高原大陆性气候, 干湿季分明, 年温差小(全 年平均气温为 -5.6 至 $\left.3.8^{\circ} \mathrm{C}\right)$, 日温差大, 日照时间 长, 辐射强烈, 无四季区分。除了少量的森林和湿 地, 草地是三江源地区分布最广泛的生态系统类 型。其中, 高寒草甸分布面积最大, 占三江源草地 面积的76\%, 其次是高寒草原(23\%)(三江源自然保 护区生态环境编辑委员会, 2002)。三江源地区生活 的大型食肉动物包括狼(Canis lupus)、棕熊(Ursus arctos)、雪豹、金钱豹(Panthera pardus)等。

\section{2 雪豹分布点收集与人类活动斑块解译}

(1)雪豹位点收集。作者于2008-2017年在三江 源全区域内, 通过粪便收集与红外相机陷阱两种方 式获得560个雪豹分布位点。在栖息地预测中, 为了 避免取样偏差和位点的空间自相关, 我们用 $1 \mathrm{~km}$ 网 格稀疏化了这些位点, 即存在于同一个 $1 \mathrm{~km}$ 网格内 的雪豹分布位点随机保留 1 个, 其他删除, 该步骤 用R代码spatialthin来处理, 最终确定 220 个位点进
入模型(图1)。

(2)人类活动信息提取。以2016年6-9月的Planet 高分辨率卫星遥感影像为底图(空间分辨率为 $5 \mathrm{~m}$ ), 通过目视解译的方法对区域内人类活动斑块进行 提取。人类活动包括农业用地、居民点、工矿用地、 采石场、能源设施、交通设施、养殖场、其他人工 设施及道路等 9 种类型。共得到人类活动斑块 16,811 处, 各级线状道路11,254处(图2)。对人类活动分布 数据进行精度评价，总体精度为 $81 \%$, Kappa系数为 0.51 , 能满足数据分析需求。

\section{3 雪豹栖息地适宜度计算}

选择29个环境变量用于拟合雪豹的栖息地适 宜度: (1)与地形相关的变量 4 个, 即海拔、坡度、坡 向、崎岖度。其中海拔来自 30 m Aster GDEM图层， 坡度、坡向与崎岖度使用海拔图层，应用ArcGIS 10.2中的空间分析工具和VRM插件计算而来; (2)增 强型植被指数(the enhanced vegetation index), 来源 于美国宇航局土地卫星装载的中分辨率成像光谱 仪MODIS; (3)土地覆盖类型，来自中国1:25万土地 覆盖遥感调查与监测数据库; (4) 19 个生物气候变 量(bioclimatic variables, Fick \& Hijmans, 2017); (5) 4 个人为影响变量, 即道路距离、距人类活动斑块距 离、道路密度和人类活动斑块密度，由此次高分辨 率卫星解译结果生成。由于19个生物气候变量的

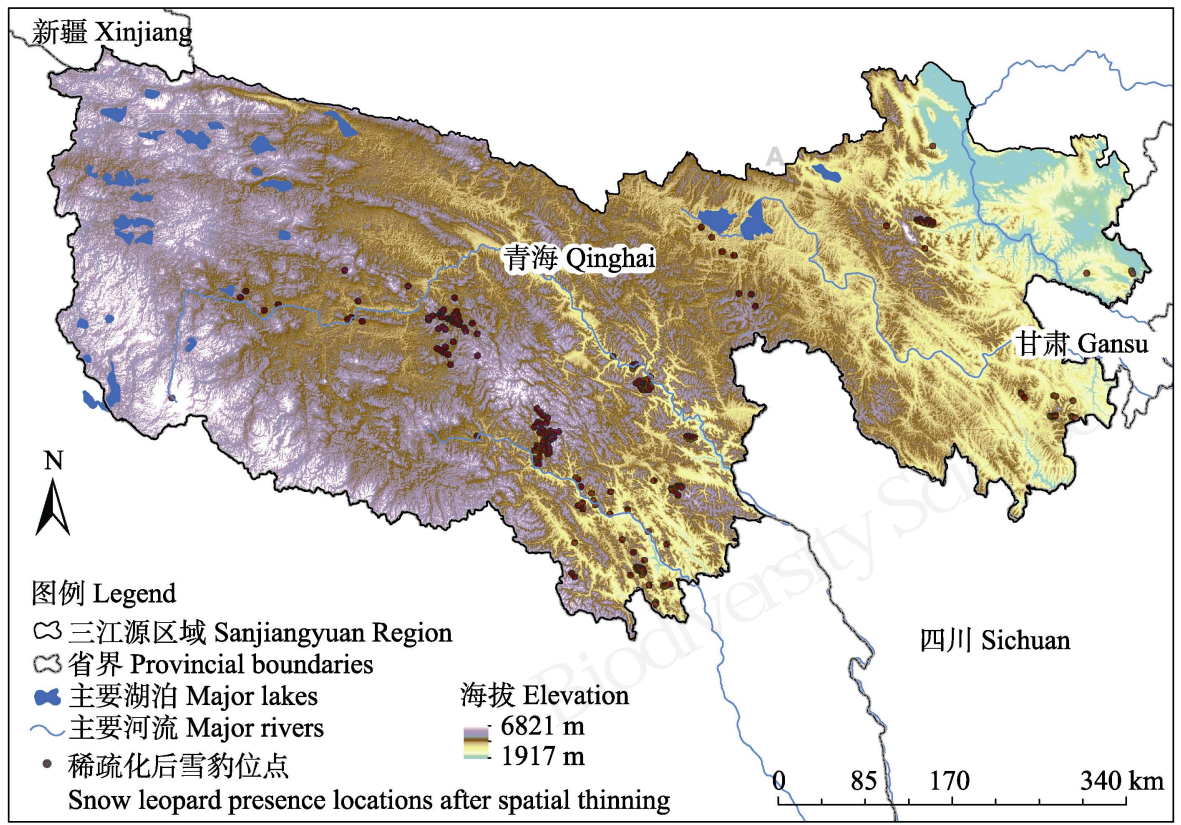

图1 三江源区域稀疏化后的 220 个雪豹出现位点

Fig. 1 The 220 presence locations of snow leopards after spatial thinning in Sanjiangyuan Region 

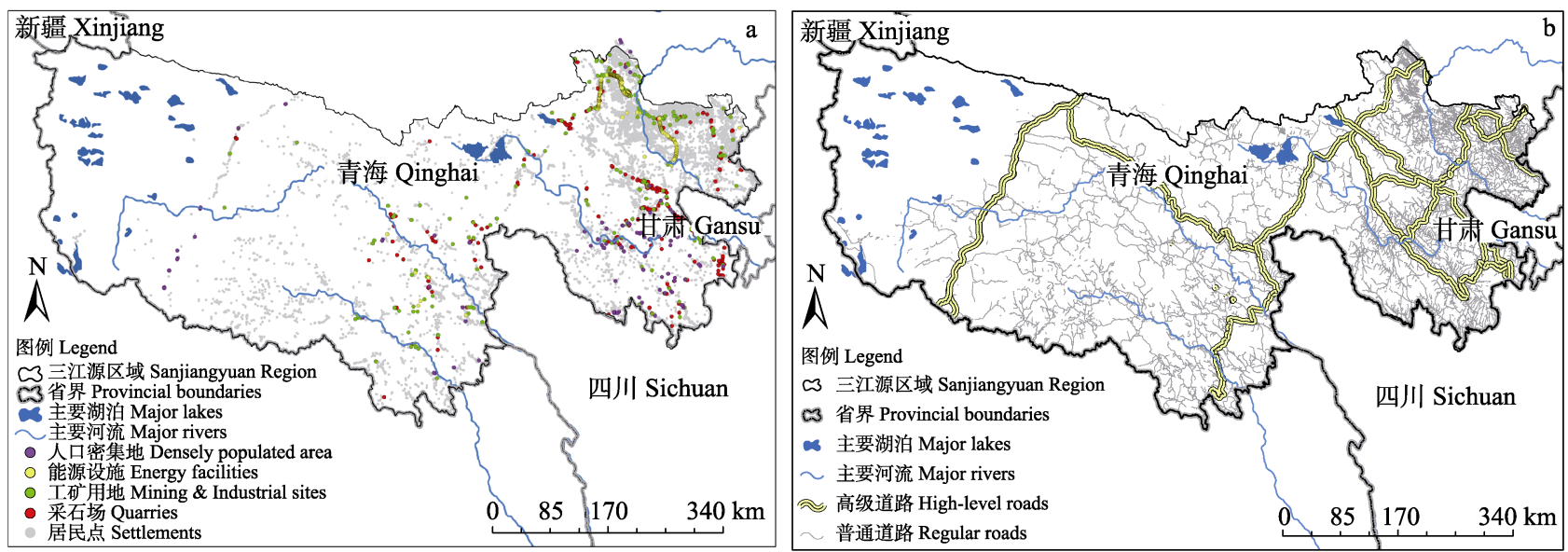

图2 三江源区域各类型人类活动斑块(a)和道路(b)的分布。此次分析中主要关注人口密集地、能源设施、工矿用地、采石场 和农村居民点这几种类型, 其中人口密集地包括城镇、机场和学校。道路被分为普通道路和高级道路两种大类, 其中高级道 路包括高速公路、国道、省道和铁路。

Fig. 2 The distribution of human activity patches (a) and roads (b) in Sanjiangyuan Region. This study mainly concerned on densely populated areas (cities and towns, airports and schools), energy facilities, mining and industry land, quarry and scattered rural settlements. Roads were classified as ordinary roads and busy roads (expressways, national highways, provincial highways and railways).

分辨率最高为 $30 \mathrm{~s}$ (约 $874 \mathrm{~m}$ ), 我们将所有变量都用 重采样的方法降到了同样的分辨率。

利用MaxEnt模型模拟三江源雪豹栖息地的分 布。在分析中，使用 $75 \%$ 的数据作为训练数据, $25 \%$ 的数据作为检验数据, 5 次重复拟合并取平均值, 用 ROC曲线检验最终的模型拟合优度 (Phillips et al, 2006)。我们选取了模型特异度与敏感度之和最大时 的值为阈值(Manel et al, 2001), 用ArcMap中的重分 类功能将此阈值以上的区域定义为雪豹适宜栖息 地。

\section{4 雪豹栖息地质量排序}

利用Zonation V4保护规划模型软件(Lehtomäki \& Moilanen, 2013)逐步将对整体保护价值贡献最低 的栖息地栅格移除，按照被移除的顺序给栖息地排 序(Moilanen et al, 2009), 划分出保护的优先等级。 采用核心区域法(Core Area Zonation)作为移除标准, 即优先保留适宜度更高的栅格。由于雪豹迁徙能力 较强, 在分区时, 通过设置30 km (雪豹最长日迁徙 距离, McCarthy et al, 2005)的平滑值来减少斑块的 破碎化, 并勾选Edge removal选项, 使其优先从边 缘开始移除，来减少保护的边缘效应。

\section{5 雪豹栖息地连通度}

利用考虑了人类活动影响的雪豹核心栖息地 图层和影响雪豹迁徙的环境阻力图层, 应用基于电 路理论设计的ArcGIS插件Linkage Mapper (V1.10),
计算核心栖息地斑块间雪豹栖息地的连通性，以及 核心栖息地的中心地位排序(与此斑块连接的斑块 越多中心地位越高)。其中环境阻力图层主要考虑以 下两个方面:

(1)自然迁徙阻力。计算公式为: 自然迁徙阻力 $=1-$ 自然栖息地适宜度

(2)人为迁徙阻力, 即人类活动带来的阻力。通 过以下方法计算, 其中 4 种影响变量的权重根据本 团队长期调查工作所积累的三江源地区各类人为 活动干扰强度的认识和经验设定：

人为迁徙阻力 $=$ 普通道路密度 $+4 \times$ 高级道 路(包括国道、省道、高速、铁路)密度 + 农村居住 点密度 $+2 \times$ 采石场密度 $+4 \times$ 工矿用地密度 + $4 \times$ 人口密集地(包括城镇、学校、机场)密度

总阻力 $=1+40 \times$ 自然迁徙阻力 $\times$ 人为迁徙 阻力, 将两种阻力相结合, 乘以 40 是为了得到数值 在1-100之间的环境阻力图层, 输入模型进行连通 度的计算。

\section{结果与分析}

\section{1 雪豹栖息地适宜度计算与潜在栖息地分布}

ROC曲线表明, 雪豹栖息地预测模型的训练数 据和检验数据的AUC值分别为 0.9244 和 0.8925 , 有 较高的模型拟合优度。Jackknife检验结果显示，对 模型综合贡献最大的因子为道路密度(17.2\%)和坡 
度(16.5\%)。其中坡度在单独使用时的独立贡献最大， 可见其带有重要的预测信息; 而道路密度在不使用 时导致的模型损失最大, 说明其带有其他变量不具 备的独立信息。从这两个主要变量的响应曲线来看, 雪豹主要生活在坡度较大、道路密度较低的区域。 根据模型特异度与敏感度之和最大所决定的阈值 重分类后，三江源地区潜在雪豹栖息地面积为 $116,570 \mathrm{~km}^{2}$ ，占整个三江源面积的31.8\% (图3)。加 入人类影响变量后模型预测的雪豹栖息地面积较 不加人类影响时减少了 2.8 万 $\mathrm{km}^{2}$, 占雪豹潜在栖息 地面积的19\%。
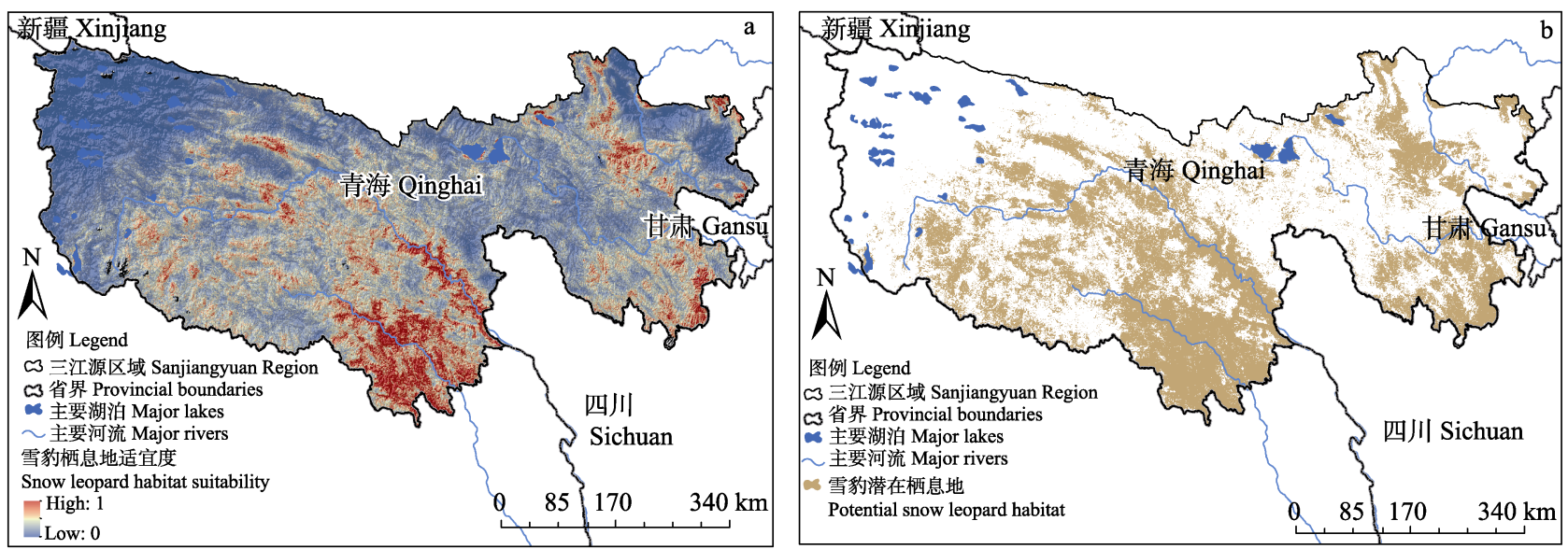

图3 三江源区域雪豹栖息地适宜度(a)和潜在栖息地分布(b)

Fig. 3 Snow leopard habitat suitability (a) and potential habitat (b) in Sanjiangyuan Region

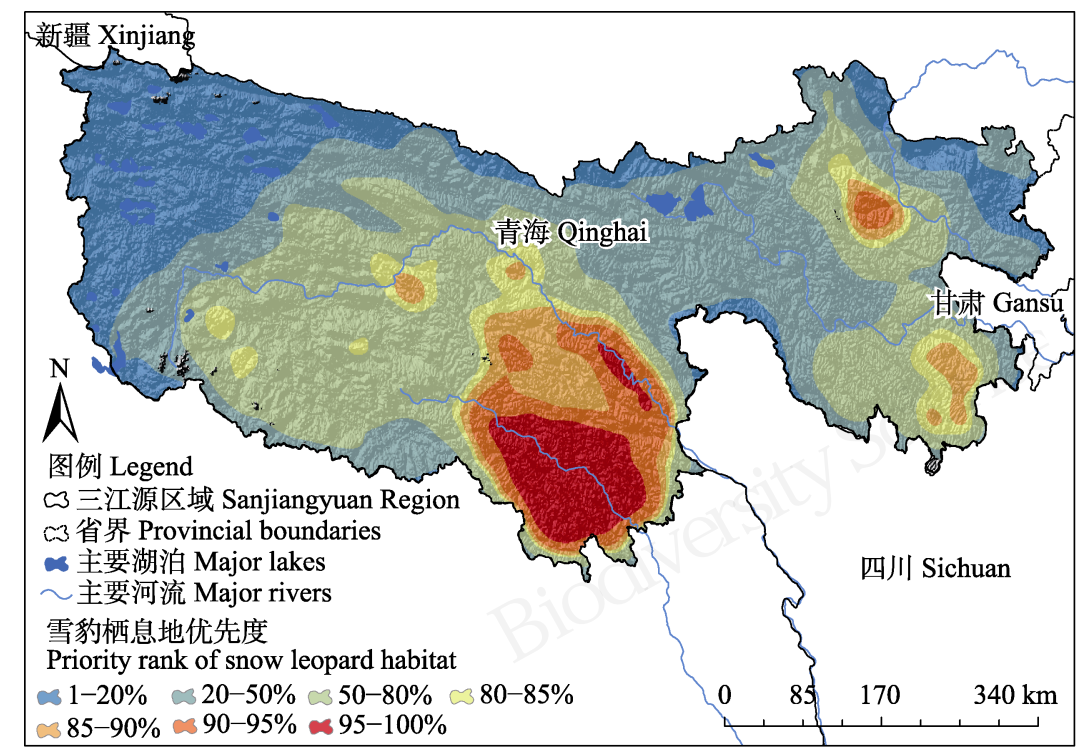

图4 三江源的雪豹栖息地质量优先度排序

Fig. 4 Priority ranking of snow leopard habitat in Sanjiangyuan Region

\section{2 雪豹核心栖息地}

根据Zonation栖息地质量排序，雪豹核心栖息 地由西向东主要分布在(图4中雪豹栖息地优先度排 序在 $80 \%-100 \%$ 区间的色块): 唐古拉山乡的两块区 域、澜沧江源头杂多县与治多县交界处的一块、治 多县索加乡、玉树-杂多-囊谦的大片连续核心栖息 地、阿尼玛卿神山区(兴海-玛沁)、年保玉则神山区 (久治-班玛), 这个结果与我们长期在三江源野外调 查的经验基本相符。加入人类影响后东部人口密集 区雪豹的核心栖息地面积缩减，西部人口稀疏区核 心栖息地面积增加(图5)。 


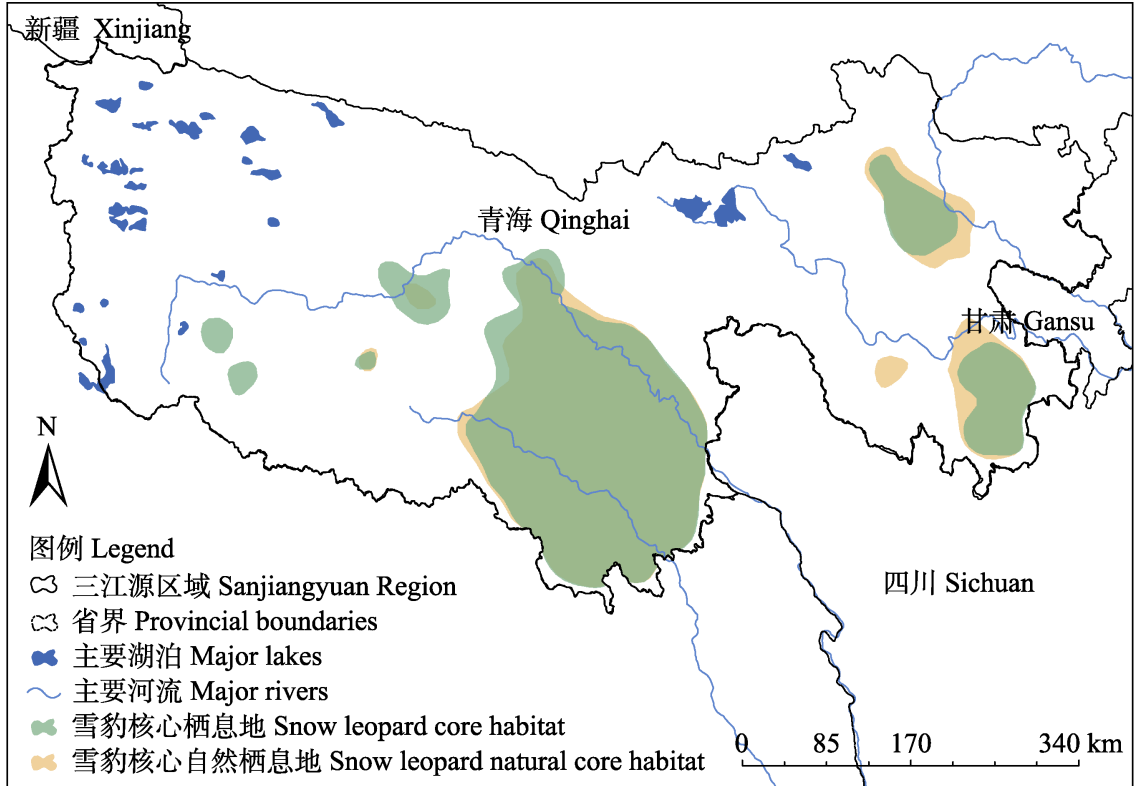

图5 加入人类影响前(黄色)后(绿色)雪豹核心栖息地的变化

Fig. 5 The change of snow leopard core habitat before (yellow) and after (green) adding anthropogenic variables

\section{3 雪豹核心栖息地之间的连通度与人类活动威} 胁评估

经过连通度分析和各核心栖息地的连通中心 地位排序, 发现玉树-杂多-囊谦这片核心栖息地不 但面积最大，且连接周边区域的中心地位也最高。 西部地区的雪豹廊道没有特别明显的窄点(pinch- point), 且迁徙廊道数量较多。其中青藏公路/铁路可 能会是唐古拉山乡的两小片核心栖息地与中心斑 块连接的潜在阻碍。东部的廊道则窄点较多, 与东 部人类活动强度较高有关, 尤其是阿尼玛卿和年保 玉则种群之间的廊道有个明显的窄点, 在甘德县的 一条省道处被拦腰截断(图6)。

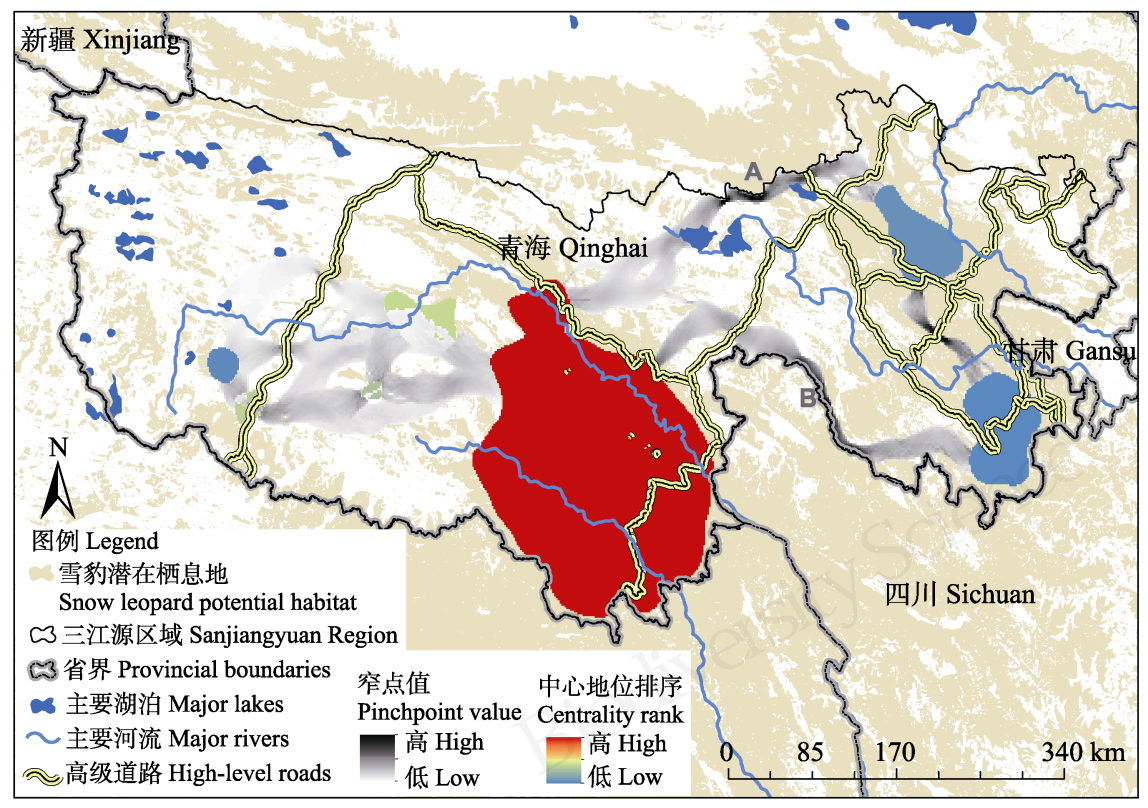

图6 雪豹核心栖息地的中心地位排序、连通度窄点(pinchpoint)和可能阻碍(高级道路)。A、B: 连接东部两个核心栖息地与 中部核心栖息地的两条可能廊道。

Fig. 6 The centrality of snow leopard core habitat, connectivity pinchpoints and busy roads as potential barriers. A, B: Two candidate corridors linking two eastern core habitats and the central core habitat. 
叠加保护区和国家公园图层与人类活动中大 型建设开发(主要包括能源设施、工矿用地和采石场) 的斑块后, 发现三江源国家级自然保护区以及新成 立的三江源国家公园均未能有效覆盖所有雪豹核 心栖息地。叠加解译出的开发活动斑块(主要包括能 源设施、工矿用地和采石场)基本都处于国家公园和 保护区之外(图7), 因此处于法定保护地外的雪豹核 心栖息地是未来需要重点监控的区域。

\section{3 不足之处与保护建议}

受到解译范围的限制，本次分析也存在一些局 限性。最主要体现在连接东部两个核心栖息地与中 部栖息地的两条廊道A, B (图6)上。如果仅仅基于三 江源内部数据, 这是迁徙阻力最小的两条路径。然 而如果叠加全国栖息地, 可以看到年保玉则种群与 玉树-杂多-囊谦种群实际上是通过西藏-四川的大 片栖息地连接的，而阿尼玛卿种群与玉树-杂多-囊 谦种群, 则更有可能是通过北部的昆仑山脉连接 (图6)。结果的局限性更说明了将此项目扩展到全国 雪豹栖息地的必要性。可以预见的是, 在新疆、甘 肃、四川等人类干扰强度更大的省份，雪豹的栖息
地分布和迁徙路径受到人类活动的影响将更为显 著，对威胁分析和保护实践的指导意义也会更大。

核心栖息地之间的连通度分析结果显示，大型 道路可能是现存阻碍雪豹迁徙最大的因素。青藏公 路/铁路可能是唐古拉山乡的两小片核心栖息地与 中心斑块连接的潜在阻碍; 阿尼玛卿和年保玉则种 群之间的廊道有一个明显的窄点，在甘德县的一条 省道处被拦腰截断。目前尚未有针对公路/铁路对雪 豹迁徙影响的研究, 未来需要设计开展青藏公路等 大型道路两侧种群基因交流及其受道路影响的针 对性研究。同时也需要开展一些针对性的保护干涉, 例如甘德县的省道周边如要加设围栏，需要考虑给 雪豹预留迁徙通道。

基于以上分析，提出针对三江源不同区域的保 护建议: (1)三江源西部核心栖息地比较小而破碎, 但迁徙通道较多且没有明显窄点，未来应关注青藏 铁路的潜在阻碍作用，同时要防范道路沿线的野生 动物盗猎。(2)中部区域横跨玉树-杂多-囊谦的雪豹 栖息地是三江源最大的核心雪豹栖息地，在与其他 种群连通中也处于中心地位，应通过种群监测确定 其健康稳定，对开发、偷猎等威胁防微杜渐，保持

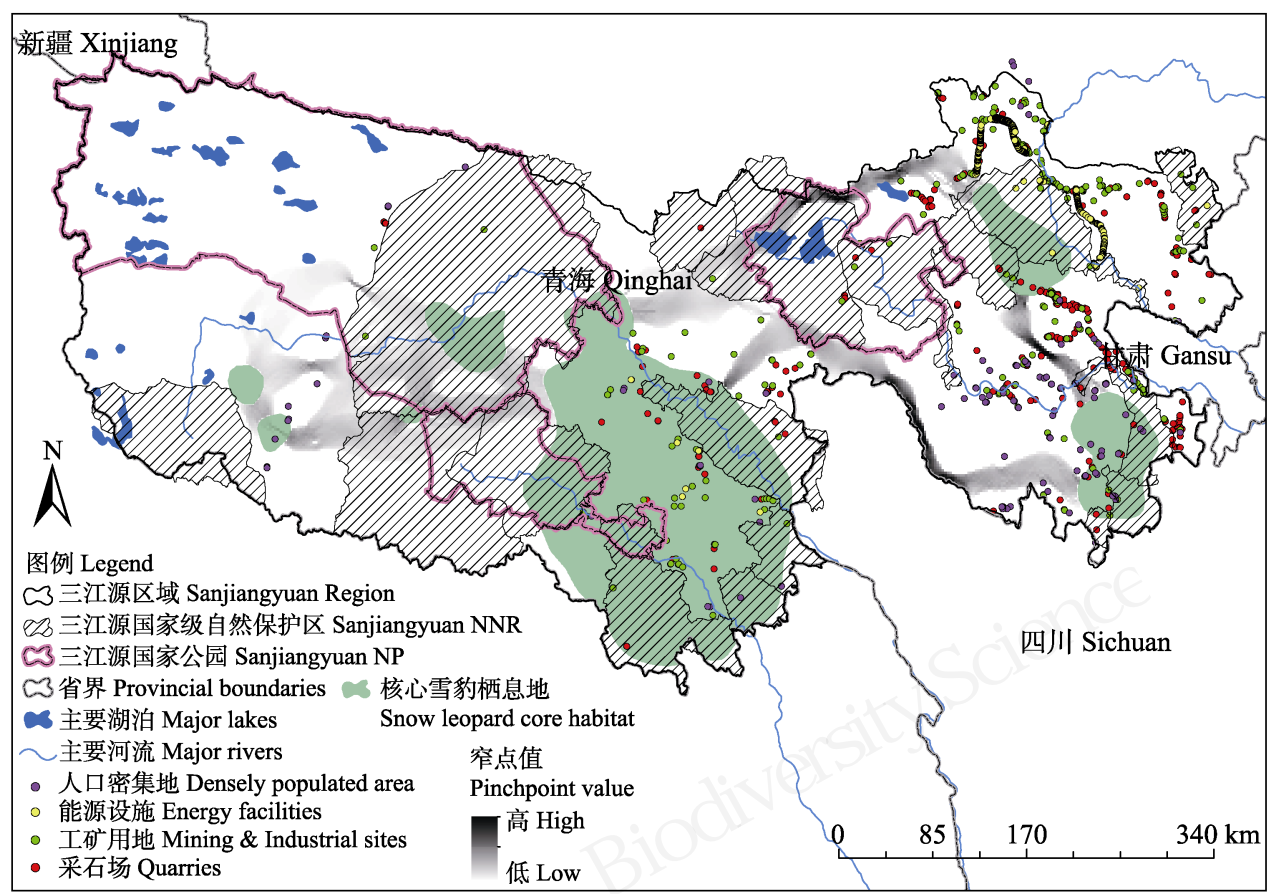

图7 国家级自然保护区与国家公园对雪豹核心栖息地的覆盖。开发项目基本存在于保护区与国家公园之外，是未来需要重 点监控开发风险的区域。

Fig. 7 The coverage of national nature reserves (NNR) and national park (NP) in snow leopard core habitat. Development projects are mostly outside nature reserves and national park, which should be intensively monitored with high-resolution satellite in the future. 
其源种群的作用。(3)东部区域人口密度高, 受人类 活动的影响最大, 应保持阿尼玛卿、年保玉则两块 核心栖息地的质量, 并重点监测甘德县境内的省道 处雪豹的迁徙通道是否畅通。

另外本次解译获得的三江源地区人类活动结 果还显示, 在雪豹的核心栖息地范围内有大量的大 型开发建设活动, 且位于法定保护地之外, 未受到 法律的保护。因此在三江源区域, 未来需重点监管 目前自然保护区与国家公园均未覆盖的物种重要 栖息地, 建议充分利用天地一体化监测手段, 开展 重要保护物种栖息地状况的评估和预警, 加强对非 保护地区域内开发建设活动的监测, 预警其对物种 保护带来的风险。

\section{参考文献}

Carbone C, Mace GM, Roberts SC, MacDonald DW (1999) Energetic constraints on the diet of terrestrial carnivores. Nature, 402, 286-288.

Cardillo M, Purvis A, Sechrest W, Gittleman JL, Bielby J, Mace GM (2004) Human population density and extinction risk in the world's carnivores. PLoS Biology, 2, e197.

Cardillo M, Mace GM, Jones KE, Bielby J, Bininda-Emonds ORP, Sechrest W, Orme CDL, Purvis A (2005) Multiple causes of high extinction risk in large mammal species. Science, 309, 1239-1241.

Davis BW, Li G, Murphy WJ (2010) Supermatrix and species tree methods resolve phylogenetic relationships within the big cats, Panthera (Carnivora: Felidae). Molecular Phylogenetics and Evolution, 56, 64-76.

Deng T, Wang XM, Fortelius M, Li Q, Wang Y, Tseng ZJ, Takeuchi GT, Saylor JE, Säilä LK, Xie GP (2011) Out of Tibet: Pliocene woolly rhino suggests high-plateau origin of Ice Age megaherbivores. Science, 333, 1285-1288.

Editorial Committee of Ecological Environment of Sanjiangyuan Nature Reserve (2002) Ecological Environment of
Sanjiangyuan Nature Reserve. Qinghai People’s Publishing House, Xining. (in Chinese) [ 《三江源自然保护区生态环 境》编辑委员会 (2002) 三江源自然保护区生态环境. 青海人民出版社, 西宁.]

Fick SE, Hijmans RJ (2017) WorldClim 2: New 1-km spatial resolution climate surfaces for global land areas. International Journal of Climatology, 37, 4302-4315.

Jackson RM, Mallon D, Sharma RK, Suryawanshi KS, Mishra C (2013) Snow Leopard Survival Strategy, Version 2013.1. Snow Leopard Network, Seattle, USA.

Lehtomäki J, Moilanen A (2013) Methods and workflow for spatial conservation prioritization using Zonation. Environmental Modelling \& Software, 47, 128-137.

Manel S, Williams HC, Ormerod SJ (2001) Evaluating presence-absence models in ecology: The need to account for prevalence. Journal of Applied Ecology, 38, 921-931.

McCarthy TM, Chapron G (2003) Snow Leopard Survival Strategy. International Snow Leopard Trust and Snow Leopard Network, Seattle, USA.

McCarthy TM, Fuller TK, Munkhtsog B (2005) Movements and activities of snow leopards in southwestern Mongolia. Biological Conservation, 124, 527-537.

McCarthy T, Mallon D, Jackson R, Zahler P, McCarthy K (2017) Panthera uncia. The IUCN Red List of Threatened Species 2017.

Moilanen A, Kujala H, Leathwick JR (2009) The Zonation framework and software for conservation prioritization. Spatial Conservation Prioritization, 135, 196-210.

Phillips SJ, Anderson RP, Schapire RE (2006) Maximum entropy modeling of species geographic distributions. Ecological Modelling, 190, 231-259.

Ripple WJ, Estes JA, Beschta RL, Wilmers CC, Ritchie EG, Hebblewhite M, Berger J, Elmhagen B, Letnic M, Nelson MP, Schmitz OJ, Smith DW, Wallach AD, Wirsing AJ (2014) Status and ecological effects of the world's largest carnivores. Science, 343, 1241484.

(责任编委: 蒋志刚 责任编辑: 间文杰) 